\title{
Probing deformed commutators with micro- and nano-oscillators
}

\author{
Mateusz Bawaj $^{1,2}$, Ciro Biancofiore ${ }^{1,2}$, Michele Bonaldi ${ }^{3,4}$, Federica Bonfigli ${ }^{1,2}$, \\ Antonio Borrielli ${ }^{3,4}$, Giovanni Di Giuseppe ${ }^{1,2}$, Lorenzo Marconi ${ }^{5}$, Francesco Marino ${ }^{6,7}$, \\ Riccardo Natali ${ }^{1,2}$, Antonio Pontin ${ }^{5,6}$, Giovanni A. Prodi ${ }^{4,8}$, Enrico Serra ${ }^{4,8,9,10}$, \\ David Vitali ${ }^{1,2}$, Francesco Marin ${ }^{5,6,11, \star}$ \\ 1 School of Science and Technology, Physics Division, \\ University of Camerino, via Madonna delle Carceri, 9, I-62032 Camerino (MC), Italy \\ ${ }^{2}$ Istituto Nazionale di Fisica Nucleare (INFN), Sezione di Perugia, Italy \\ ${ }^{3}$ Institute of Materials for Electronics and Magnetism, \\ Nanoscience-Trento-FBK Division, I-38123 Povo (TN), Italy \\ ${ }^{4}$ INFN, Trento Institute for Fundamental Physics and Application, I-38123 Povo (TN), Italy \\ ${ }^{5}$ Dipartimento di Fisica e Astronomia, Università di Firenze, \\ via Sansone 1, I-50019 Sesto Fiorentino (FI), Italy \\ ${ }^{6}$ INFN, Sezione di Firenze, via Sansone 1, I-50019 Sesto Fiorentino (FI), Italy \\ 7 CNR-Istituto Nazionale di Ottica, largo E. Fermi 6, I-50125 Firenze, Italy \\ 8 Dipartimento di Fisica, Università di Trento, I-38123 Povo (TN), Italy \\ 9 Centre for Materials and Microsystem, Fondazione Bruno Kessler, I-38123 Povo (TN), Italy \\ 10 Dept. of Microelectronics and Computer/ECTM/DIMES Technology Centre, Delft University \\ of Technology, Feldmanweg 17, 2628 CT Delft, PO Box 5053, 2600 GB Delft, The Netherlands \\ 11 European Laboratory for Non-Linear Spectroscopy (LENS), \\ via Carrara 1, I-50019 Sesto Fiorentino (FI), Italy \\ *e-mail: marin@fi.infn.it
}

\begin{abstract}
A minimal observable length is a common feature of theories that aim to merge quantum physics and gravity. Quantum mechanically, this concept is associated to a minimal uncertainty in position measurements, which is encoded in deformed commutation relations. Once applied in the Heisenberg dynamics, they give effects potentially detectable in low energy experiments. For instance, an isolated harmonic oscillator becomes intrinsically nonlinear and its dynamics shows a dependence of the oscillation frequency on the amplitude, as well as the appearance of higher harmonics. Here we analyze the free decay of micro and nano-oscillators, spanning a wide range of masses, and we place upper limits to the parameters quantifying the commutator deformation.
\end{abstract}

Keywords: Planck scale; modified uncertainty relations; mechanical oscillator.

\section{Introduction and model}

General relativity and quantum physics are expected to merge at the Planck scale, defined by distances of the order of $L_{\mathrm{P}}=1.6 \times 10^{-35} \mathrm{~m}$ and/or extremely high energies of the order of $E_{\mathrm{P}}=1.2 \times 10^{19} \mathrm{GeV}$. Since the study of particles collisions around the Planck energy is well beyond the possibilities of current and foreseeable accelerators, high-energy astronomical events (e.g. $\gamma$-ray bursts) have been considered as the privileged natural system to unveil quantum gravitational effects. This common view has been enriched in the last years thanks to a number of studies proposing that signatures of the Planck-scale physics could manifest also at low energies ${ }^{1-5}$. It is indeed widely accepted that a re-formulation of quantum theory should be required to incorporate gravitational effects at the Planck scale. In particular, gravity induces an additional quantum uncertainty, which is typically 
encoded into deformed commutation relations between position $q$ and momentum $p^{6-8}$ :

$$
[q, p]=i \hbar\left(1+\beta_{0}\left(\frac{L_{\mathrm{P} p}}{\hbar}\right)^{2}\right) .
$$

In this framework, the dynamics of a harmonic oscillator becomes intrinsically nonlinear and shows an unusual dependence of the oscillation frequency on the amplitude. Here we describe an experiment conceived to test this hypothesis, and set limits to the deformation parameter $\beta_{0}{ }^{9}$. Our work is based on two assumptions. First, we suppose that Eq. (1) holds for the operator $q$ describing a measured position in a macroscopic harmonic oscillator, and its conjugate momentum $p$. In terms of the usually normalized operators, $Q=q / \sqrt{\hbar /\left(m \omega_{0}\right)}$ and $P=p / \sqrt{\hbar m \omega_{0}}$, defined for for an oscillator with mass $m$ and resonance angular frequency $\omega_{0}$, the commutation relations are therefore

$$
[Q, P]=i\left(1+\beta P^{2}\right),
$$

where $\beta=\beta_{0}\left(\hbar m \omega_{0} / m_{\mathrm{P}}^{2} c^{2}\right)\left(m_{\mathrm{P}}=E_{\mathrm{P}} / c^{2}\right.$ is the Planck mass $)$ is a further dimensionless parameter that we assume to be small $(\beta \ll 1)$. Such assumption will be shown to be consistent with the experimental results. The second hypothesis is the validity of the Heisenberg equations for the temporal evolution of an operator $\hat{O}$, i.e. $\mathrm{d} \hat{O} / \mathrm{d} t=[\hat{O}, H] / i \hbar$, where $H$ is the Hamiltonian $H=\frac{\hbar \omega_{0}}{2}\left(Q^{2}+P^{2}\right)$.

In particular, the standard Heisenberg evolution equations are applied to the operators $P$ and $Q$. The solution is

$$
Q=Q_{0}\left[\sin (\tilde{\omega} t)+\frac{\beta}{8} Q_{0}^{2} \sin (3 \tilde{\omega} t)\right],
$$

where

$$
\tilde{\omega}=\left(1+\frac{\beta}{2} Q_{0}^{2}\right) \omega_{0} .
$$

It is valid at the first order in $\beta Q_{0}^{2}$, and implies two relevant effects with respect to the harmonic oscillator: a) the appearance of the third harmonic and, less obvious, b) a quadratic dependence of the frequency shift on the oscillation amplitude. In case of small damping with relaxation time $\tau$, the dynamics is described by a modified version of Eq. (3) with the replacements $\tilde{\omega} t \rightarrow \Phi(t)$, implying $\tilde{\omega}(t)=\mathrm{d} \Phi / \mathrm{d} t$, and $Q_{0} \rightarrow Q_{0} \exp (-t / \tau)$.

\section{Experiment}

We have exploited three kinds of oscillators, spanning a wide range of masses around the Planck mass $m_{\mathrm{P}}=22 \mu \mathrm{g}$. The measurements are performed by exciting an oscillation mode and monitoring a possible dependence of the oscillation frequency and of the third harmonic distortion on the oscillation amplitude, during the free decay. The first device is a "double paddle oscillator" (DPO) ${ }^{10}$ made from a $300 \mu \mathrm{m}$ thick 
silicon plate. Thanks to its shape, for two particular balanced oscillation modes, the oscillator is supported by the outer frame with negligible energy dissipation and it can therefore be considered as isolated from the background ${ }^{11}$. The sample is kept in a temperature stabilized vacuum chamber and vibrations are excited and detected capacitively. We have monitored the mode oscillating at frequency of $5636 \mathrm{~Hz}$ with a mechanical quality factor of $1.18 \times 10^{5}$ (at room temperature) and mass $m=0.033 \mathrm{~g}$. For the measurements at intermediate mass we have used
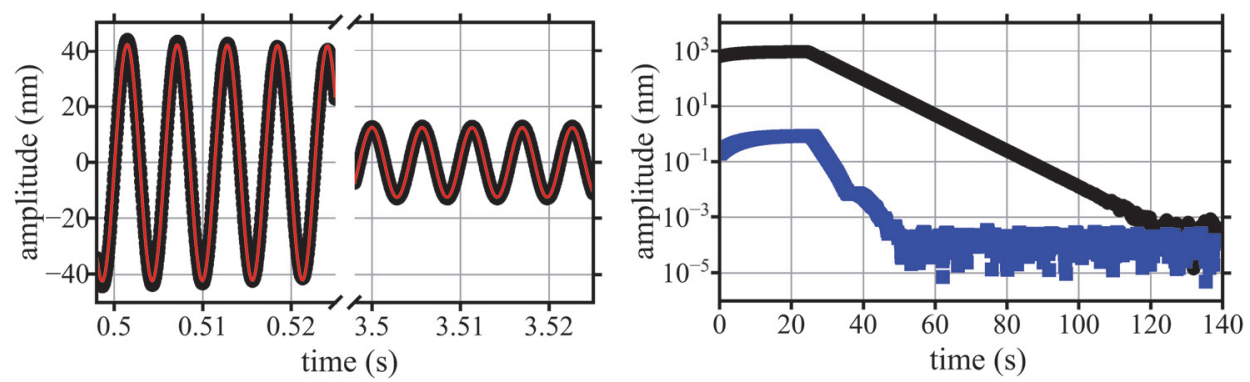

Fig. 1. a) Example of the decaying oscillation of the wheel oscillator, down shifted to $\sim 200 \mathrm{~Hz}$ by a lock-in amplifier, fitted with the theoretical model including the possibility of a parabolic dependence of the oscillation frequency on the amplitude. b) Simultaneous detection of the first and third harmonic in the decay of the DPO oscillator.

a silicon wheel oscillator, made on the $70 \mu \mathrm{m}$ thick device layer of a SOI wafer and composed of a central disk kept by structured beams ${ }^{12}$. The disk motion is balanced by four counterweights on the beams joints that so become nodal points (Fig. 1b ${ }^{13}$. On the surface of the central disk, a multilayer $\mathrm{SiO}_{2} / \mathrm{Ta}_{2} \mathrm{O}_{5}$ dielectric coating forms an high reflectivity mirror. The design strategy allows to obtain a balanced oscillating mode (its resonance frequency is $141797 \mathrm{~Hz}$ ), with a planar motion of the central mass (significantly reducing the contribution of the optical coating to the structural dissipation) and a strong isolation from the frame. The oscillator is mechanically excited using a piezoelectric ceramic glued on the sample mount. The surface of the core of the device works as end mirror in one arm of a stabilized Michelson interferometer, that allows to measure its displacement. The measurements are performed at the temperature of $4.3 \mathrm{~K}$, where the mechanical quality factor surpasses $10^{6}$ and thermal noise is strongly reduced. The meaningful mass is $m=20 \mu \mathrm{g}$. Finally, the lighter oscillators is a $L=0.5 \mathrm{~mm}$ side, $30 \mathrm{~nm}$ thick, square membrane of stoichiometric silicon nitride, grown on a $5 \mathrm{~mm} \times 5 \mathrm{~mm}$, $200 \mu \mathrm{m}$ thick silicon substrate ${ }^{14}$. The physical mass of the membrane is $20 \mathrm{ng}$. We have performed the measurements in a cryostat at the temperature of $65 \mathrm{~K}$ and pressure of $10^{-4} \mathrm{~Pa}$, where the oscillation frequency is $747 \mathrm{kHz}$ and the quality factor is $8.6 \times 10^{5}$. Excitation and readout are performed as in the experiment with the wheel oscillators. 
The frequency shift of the oscillation as function of the amplitude is obtained both by directly fitting the exponentially decaying oscillation with the expected expression, in some cases after a preliminary frequency down-conversion performed with a lock-in amplifier (see Fig. 1a for an example), and by completely frequency down-converting the oscillating signal (down to dc) with hardware and software lock-in amplifiers, then calculating the frequency as first derivative of the residual phase. Similarly, the third harmonic content is deduced both from the fit of time series, and by simultaneous recording of first an third harmonic of the signal with two separated lock-in amplifiers (Fig. 1b). For both indicators, the two methods give comparable results.

Table 1. Maximum relative frequency shifts measured for different oscillators, and corresponding oscillation amplitudes.

\begin{tabular}{|ccccc|}
\hline $\begin{array}{c}\text { Mass } \\
(\mathrm{kg})\end{array}$ & $\begin{array}{c}\text { Frequency } \\
(\mathrm{Hz})\end{array}$ & $\begin{array}{c}\text { Max. ampl. } \\
(\mathrm{nm})\end{array}$ & $1 / Q$ & Max. $\Delta \omega / \omega_{0}$ \\
\hline $3.3 \times 10^{-5}$ & $5.64 \times 10^{3}$ & 600 & $8 \times 10^{-6}$ & $4 \times 10^{-7}$ \\
$2 \times 10^{-8}$ & $1.42 \times 10^{5}$ & 55 & $1 \times 10^{-6}$ & $6 \times 10^{-8}$ \\
$2 \times 10^{-11}$ & $7.47 \times 10^{5}$ & 47 & $1.2 \times 10^{-6}$ & $3 \times 10^{-6}$ \\
\hline
\end{tabular}

We remark that the frequency spread of the oscillating signal is typically lower than the linewidth, as shown in Table 1 . We note that $\Delta \omega / \omega_{0} \ll 1$ is required in order for the model to be valid (indeed, it implies $\beta Q_{0}^{2} \ll 1$ ).

In all cases, for large enough excitation, the frequency shows indeed a parabolic dependence on the oscillation amplitude. This feature can be attributed to structural nonlinearity which is intrinsic in all the oscillators. The interesting upper limits are obtained by summing the measured parabolic coefficient and its $95 \%$ uncertainty, extracting the corresponding $\beta$ from Eq. (4), and finally calculating $\beta_{0}$. A similar procedure is used for the third harmonic contents. A detailed modelling of the structural nonlinearity could allow to subtract its effects from the data, and thus place even stronger limits to the residual nonlinearity and actually to $\beta_{0}$. However, a quantitative prediction of such effects is extremely challenging, as it strongly depends also on the geometry of specific oscillator. Therefore, the above results can be considered as the state of the art measurements in such systems.

\section{Results and discussion}

Our results are summarized in Fig. 2, where we also report some previously existing limits to the deformation parameter $\beta_{0}$. We have achieved a significant improvement, by many orders of magnitude, working on different oscillators and analyzing different physical observables. In our experiments we have considered a wide range of masses around the Planck mass. We believe our analysis to be particularly meaningful in this regime, as strong deviations from classical Newtonian mechanics arise as soon as the momentum is of the order of $m_{P} c$. This is not only true for planetary motion, but even for Kg-scale mechanical oscillators. In these cases, the lack 


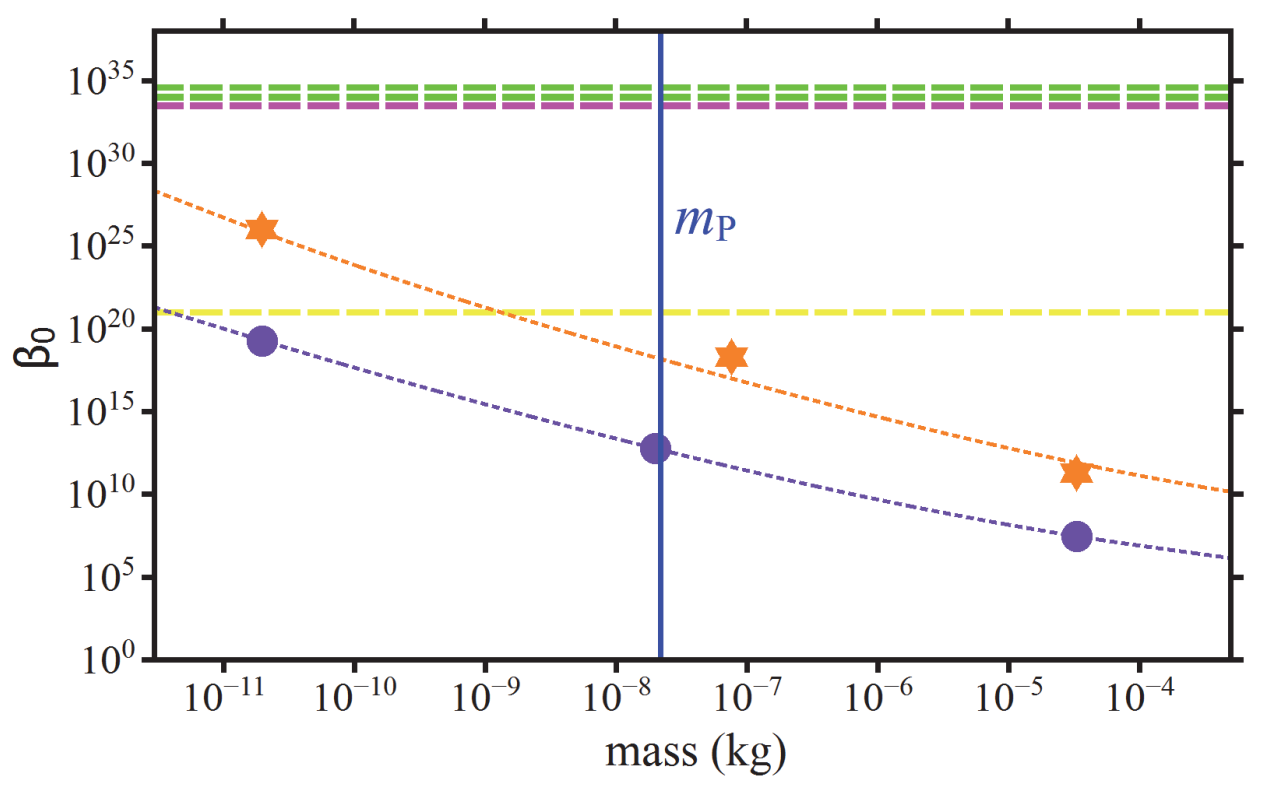

Fig. 2. The parameter $\beta_{0}$ quantifies the deformation to the standard commutator between position and momentum, or the scale $\sqrt{\beta_{0}} L_{\mathrm{P}}$ below which new physics could come into play. Full symbols report its upper limits obtained in this work, as a function of the mass. Blue dots: from the amplitude-dependence of the oscillation frequency; red stars: from the third harmonic distortion. Dotted lines are guides for the eyes. Dashed lines reports some previously estimated upper limits, obtained in mass ranges outside this graph. At lower mass, in green: from high resolution spectroscopy on the hydrogen atom, considering the ground state Lamb shift (upper line) ${ }^{2}$ and the 1S-2S level difference (lower line) ${ }^{1}$. At higher mass, in magenta: from the AURIGA detector ${ }^{4,5}$, in yellow: from the lack of violation of the equivalence principle ${ }^{15}$.

of observable deviations would imply the need to revise the rules connecting quantum to classical dynamics, or it could indicate a possible mass dependence of the deformation parameter. In this context, our experimental measurements should not be simply intended as a check of possible deformations of quantum mechanics, but as a test of a "composite" hypothesis, involving also the form of the classical limit corresponding to the modified quantum rules. We remark indeed that the present approach involves just the expectation values of position and momentum operators. Any theory predicting the modifications of such quantities would unavoidably imply also deviations from the classical dynamics. A more subtle route to the search of quantum gravitational effects could instead focus on specific quantum features of a system. For instance, quantum fluctuations of the spacetime metric and/or spacetime discreteness are expected to significantly affect the evolution of higher order momenta. On the other hand, it cannot be excluded that more refined models could include some protection against trivial (and potentially catastrophic) modifications of classical dynamics even for the simple expectation values. For instance, such modifications could be destroyed by the interaction with the thermal bath, i.e., by 
the decoherence of the wavefunction. This motivates future experiments, based on quantum macroscopic oscillators, i.e., by oscillators previously cooled down to an average thermal occupation number below unity.

\section{References}

1. C. Quesne, and V.M. Tkachuk, Phys. Rev. A 81, 012106 (2010).

2. A.F. Ali, S. Das, and E.C. Vagenas, Phys. Rev. D 84, 044013 (2011).

3. I. Pikovski et al., Nature Phys. 8, 393 (2012).

4. F. Marin et al., Nature Phys. 9, 71 (2013).

5. F. Marin et al., New J. Phys. 16, 085012 (2014).

6. D. Amati, M. Ciafaloni, and G. Veneziano, Phys. Lett. B 197, 81 (1987).

7. D.J. Gross, and P.F. Mende, Nucl. Phys. B 303, 407 (1988).

8. L.G. Garay, et al., Int. J. Mod. Phys. A 10, 145 (1995).

9. M. Bawaj et al., Nature Communications 6, 7503 (2015).

10. C.L. Spiel, R.O. Pohl, and A.T. Zehnder, Rev. Sci. Inst. 72, 1482 (2001).

11. A. Borrielli et al., J. Micromech. Microeng. 21, 065019 (2011).

12. E. Serra et al., Appl. Phys. Lett. 101, 071101 (2012).

13. A. Borrielli et al., Microsyst. Technol. 20, 907 (2014).

14. J.D. Thompson et al. Nature 452, 72 (2008).

15. S. Ghosh, Class. Quantum Grav. 31, 025025 (2014). 